\title{
Atrium-targeted drug delivery through an amiodarone-eluting bilayered patch
}

\author{
Robert W. Bolderman, MD, ${ }^{\text {a }}$ Peter Bruin, PhD, ${ }^{c}$ J. J. Rob Hermans, PhD, ${ }^{b}$ Mark J. Boerakker, PhD, ${ }^{c}$ \\ Aylvin A. Dias, $\mathrm{PhD},{ }^{\mathrm{c}}$ Frederik H. van der Veen, $\mathrm{PhD},{ }^{\mathrm{a}}$ and Jos G. Maessen, MD, $\mathrm{PhD}^{\mathrm{a}}$
}

\begin{abstract}
Objective: Clinical studies have demonstrated the efficacy of oral and intravenous amiodarone therapy to prevent postoperative atrial fibrillation. However, because of significant extracardiac side effects, only high-risk patients are eligible for prophylactic amiodarone therapy. This study addressed the hypothesis that atrium-specific drug delivery through an amiodarone-eluting epicardial patch reduces vulnerability to atrial tachyarrhythmias, whereas ventricular and plasma drug concentrations are minimized.
\end{abstract}

\begin{abstract}
Methods: Right atrial epicardiums of goats were fitted with electrodes and a bilayered patch (poly[ethylene glycol]-based matrix and poly[lactide-co-caprolactone] backing layer) loaded with amiodarone (10 $\mathrm{mg}$ per patch, $\mathrm{n}=10$ ) or without drug $(\mathrm{n}=6)$. Electrophysiologic parameters (atrial effective refractory period, conduction time, and rapid atrial response to burst pacing) and amiodarone levels in plasma and tissue were measured during 1 month's follow-up.
\end{abstract}

Results: Epicardial application of amiodarone-eluting patches produced persistently higher drug concentrations in the right atrium than in the left atrium, ventricles, and extracardiac tissues by 2 to 4 orders of magnitude. Atrial effective refractory period and conduction time increased, whereas rapid atrial response inducibility decreased significantly $(P<.05)$ during the 1 -month follow-up compared with that seen in animals treated with drugfree patches. Amiodarone concentrations in plasma remained undetectably low $(<10 \mathrm{ng} / \mathrm{mL})$.

Conclusions: Atrium-specific drug delivery through an amiodarone-eluting patch produces therapeutic atrial drug concentrations, whereas ventricular and systemic drug levels are minimized. This study demonstrates that sustained targeted drug delivery to a specific heart chamber is feasible and might reduce the risk for ventricular and extracardiac adverse effects. Epicardial application of amiodarone-eluting patches is a promising strategy to prevent postoperative atrial fibrillation. (J Thorac Cardiovasc Surg 2010;140:904-10)

Postoperative atrial fibrillation (POAF) is the most frequent complication of cardiac surgery, with incidence rates varying between $20 \%$ and $60 \%$ depending on the type of procedure and the criteria for diagnosis. ${ }^{1} \mathrm{POAF}$ is associated with an increased risk of morbidity and mortality caused by stroke, heart failure, ventricular arrhythmias, thromboembolism, and bleeding from anticoagulation. ${ }^{2}$ Furthermore, POAF increases length of hospital stay, hospital readmission rate, and hospital costs.

According to the American College of Cardiology/American Heart Association/European Society of Cardiology

From the Departments of Cardiothoracic Surgery ${ }^{\mathrm{a}}$ and Pharmacology, ${ }^{\mathrm{b}}$ Cardiovascular Research Institute Maastricht, Maastricht, The Netherlands; and DSM Biomedical, ${ }^{\mathrm{c}}$ Geleen, The Netherlands.

Supported within the framework of the Biomaterials program, a private-public joint effort of the Dutch Ministry of Economic Affairs, the Province of Dutch Limburg, DSM Research BV (Geleen, The Netherlands), and the Maastricht University Medical Centre.

Disclosures: None.

Received for publication Nov 1, 2009; revisions received Dec 18, 2009; accepted for publication Jan 10, 2010; available ahead of print April 5, 2010.

Address for reprints: Robert W. Bolderman, MD, Cardiovascular Research Institute Maastricht, Department of Cardiothoracic Surgery, Universiteitssingel 50, PO Box 616, 6200 MD Maastricht, The Netherlands (E-mail: r.bolderman@ctc. unimaas.nl).

$0022-5223 / \$ 36.00$

Copyright (c) 2010 by The American Association for Thoracic Surgery doi:10.1016/j.jtcvs.2010.01.021
2006 joint guidelines, POAF should be prevented by treatment with an oral $\beta$-blocker or, in patients are at high risk, preoperative administration of amiodarone. ${ }^{3}$ Although amiodarone is probably the most effective drug, it is reserved as a second-line agent because of its associated risk of potentially severe extracardiac adverse effects, such as thyroid dysfunction, pulmonary toxicity, and hepatic toxicity. ${ }^{4}$ These effects are in part attributable to the relatively high drug concentrations required with systemic administration. Another disadvantage of amiodarone is the need for a loading period. ${ }^{5}$

Local drug delivery could minimize the ventricular and extracardiac adverse effects of amiodarone and could make a loading period superfluous. Several studies investigated intrapericardial infusion of amiodarone solutions to achieve local drug delivery. ${ }^{6-8}$ However, this method does not allow for atrium-specific drug targeting because the drug equally diffuses to the ventricular epicardium. Epicardial application of drug-releasing biomaterials might offer the advantage of a more localized (heart chamber-targeted) drug delivery, although diffusion to other heart chambers, partly through leakage to the pericardial fluid, is still possible. ${ }^{9}$

We reasoned that atrium-specific amiodarone therapy might be achieved if a bilayered patch would be applied epicardially in such a way that an amiodarone-loaded matrix 


$$
\begin{aligned}
& \text { Abbreviations and Acronyms } \\
& \text { ECG }=\text { electrocardiography } \\
& \text { ERP }=\text { effective refractory period } \\
& \text { LA }=\text { left atrium } \\
& \text { LV }=\text { left ventricle } \\
& \text { PEG }=\text { poly(ethylene glycol) } \\
& \text { PLCL }=\text { poly(lactide-co-caprolactone) } \\
& \text { POAF }=\text { postoperative atrial fibrillation } \\
& \text { RA }=\text { right atrium } \\
& \text { RAR }=\text { rapid atrial response } \\
& \text { RV }=\text { right ventricle }
\end{aligned}
$$

faces the epicardium and an impermeable backing layer faces the pericardium. This study addressed the hypothesis that amiodarone-eluting bilayered patches reduce vulnerability to atrial tachyarrhythmias in chronically instrumented goats while ventricular and systemic drug concentrations are minimized.

\section{MATERIALS AND METHODS}

All procedures were approved by the Animal Studies Committee of the University of Maastricht and performed according to the principles of "The guide for the care and use of laboratory animals" published by the National Institutes of Health (National Institutes of Health publication no. 85-23, revised 1996).

Domestic goats $(\mathrm{n}=16$; weight, $47-67 \mathrm{~kg}$ ) were randomly assigned to a drug-free patch group (DFP group, $\mathrm{n}=6$ ) or an amiodarone-loaded patch group (ALP group, $n=10$ ). Four animals of the ALP group were only used for pharmacokinetic studies, whereas all other animals were chronically instrumented with epicardial electrodes.

\section{Patch Synthesis}

Preparation of poly(ethylene glycol) 10,000 tetra(4pentenoate). Under a nitrogen atmosphere, $20 \mathrm{~g}$ of poly(ethylene glycol) (PEG) 10,000 4-arm hydroxyl (Nektar Therapeutics, San Carlos, Calif) was dissolved in $200 \mathrm{~mL}$ of toluene and $50 \mathrm{~mL}$ of dichloromethane and cooled with an ice bath. Triethylamine $(2.8 \mathrm{~mL})$ was added to the clear solution. Then $2.4 \mathrm{~mL}$ of 4-pentenoylchloride (Sigma-Aldrich, St Louis, Mo) was added dropwise to the ice-cooled solution. The reaction was continued overnight at room temperature. Triethylamine salts were then filtered off in vacuo, and the clear filtrate was precipitated into $2 \mathrm{~L}$ of ice-cold diethylether. The solid precipitate was collected and redissolved in $100 \mathrm{~mL}$ of chloroform. This solution was reprecipitated into excess of hexane. The precipitate was collected and dried in vacuo. This yielded approximately $15 \mathrm{~g}$ $(97.8 \%)$ of star-shaped PEG 10,000 tetra(4-pentenoate) as a white powder. The polymer was characterized by using ${ }^{1} \mathrm{H}$-nuclear magnetic resonance spectroscopy (at $300 \mathrm{MHz}, 22^{\circ} \mathrm{C}$ with deuterated chloroform as a solvent and tetramethylsilane as an internal standard): $\delta(\mathrm{ppm})=5.79(\mathrm{~m}, 4 \mathrm{H}$, $\left.-\mathrm{CH}=\mathrm{CH}_{2}\right) ; 5.05\left(\mathrm{~m}, 8 \mathrm{H},-\mathrm{CH}=\mathrm{CH}_{2}\right) ; 4.22\left(\mathrm{t}, 8 \mathrm{H},-\mathrm{OCH}_{2} \mathrm{CH}_{2} \mathrm{O}[\mathrm{C}=\mathrm{O}-)\right.$; and 3.89 to $3.35\left(\mathrm{~m}, 938 \mathrm{H},-\mathrm{OCH}_{2} \mathrm{CH}_{2} \mathrm{O}\left[\mathrm{C}=\mathrm{O}-\right.\right.$ and $\left.-\left[\mathrm{OCH}_{2} \mathrm{CH}_{2}\right]_{\mathrm{n}} \mathrm{O}-\right)$ and $\mathrm{C}\left(\mathrm{CH}_{2} \mathrm{O}\right) 4$.

Fabrication of bilayered patches. Two grams of PEG 10,000 tetra(4-pentenoate) and $0.1 \mathrm{~g}$ adipic thioic acid (1,6-hexane dithioic acid) were dissolved in $2 \mathrm{~mL}$ of tetrahydrofuran. ${ }^{10}$ Photoinitiator Darocur 1173 (0.05 g; Ciba, Basel, Switzerland) and $10 \% \mathrm{wt} / \mathrm{wt}$ (relative to PEG plus cross-linker) amiodarone powder (Sigma-Aldrich) were added to this solu- tion. The suspension was stirred vigorously and transferred into circular Teflon molds (14-mm diameter) on top of a 50- $\mu$ m-thick elastic film composed of a poly(lactide-co-caprolactone) (PLCL) copolymer. The suspensions were exposed to UV light $\left(5 \times 1 \mathrm{~J} / \mathrm{cm}^{2}\right)$ and dried in vacuo for several days, resulting in the formation of biodegradable bilayered discoidal patches composed of a amiodarone-loaded PEG matrix and a PLCL backing layer. Patches were $300 \mu \mathrm{m}$ thick, had a diameter of $13 \mathrm{~mm}$, weighed $101 \pm 4 \mathrm{mg}$, and contained $9.9 \pm 0.4 \mathrm{mg}$ of amiodarone. The amiodarone dose was selected based on pilot experiments, which demonstrated an increase in atrial effective refractory periods (ERPs) 28 days after patch application. Drug-free patches were synthesized according to the same procedure without the addition of amiodarone.

\section{Patch Application}

Goats were anesthetized with isoflurane ( $2 \%$ to $3 \%)$ with a $1: 1$ mixture of oxygen and air after induction of anesthesia with thiopental, $20 \mathrm{mg} / \mathrm{kg}$ administered intravenously. During surgical intervention, limb-lead electrocardiography (ECG), arterial blood pressure, temperature, end-expiratory $\mathrm{CO}_{2}$, and oxygen saturation (pulse oximetry) were monitored. Saline $(0.9 \%, 5-8 \mathrm{~mL} / \mathrm{kg}$ per hour $)$ was infused through a peripheral venous catheter. Buprenorphine (10 $\mu \mathrm{g} / \mathrm{kg}$ administered intravenously) was used as an analgesic.

A right intercostal thoracotomy was performed, and the right atrial lateral wall was exposed. In goats scheduled for chronic electrophysiologic studies, 2 silicone strips of $10 \times 10 \mathrm{~mm}$, each containing 4 silver unipolar electrodes (1.5-mm diameter and 5-mm interelectrode distance), were sutured to the right atrial lateral wall, one close to the superior caval vein and the other close to the inferior caval vein. After baseline measurements, a patch (loaded with or without amiodarone) was sutured to the right atrial lateral wall (between the 2 electrode-containing silicone strips), and electrophysiologic measurements were repeated after 1 hour. The pericardium and thorax were subsequently closed, and the electrode leads were tunneled subcutaneously to the neck and exteriorized. Animals were injected with prophylactic ampicillin $(20 \mathrm{mg} / \mathrm{kg}$ administered intramuscularly) before and directly after surgical intervention. Buprenorphine $(10 \mu \mathrm{g} / \mathrm{kg}$ administered intramuscularly twice daily) was administered postoperatively for 3 days.

\section{Electrophysiologic Studies}

Electrophysiologic parameters were measured during surgical intervention and at days $1,2,3,4,7,9,11,14,16,18,21,23,25$, and 28 after surgical intervention. ECG limb leads and electrodes were connected to an electrophysiologic monitoring system with an integrated amplifier/stimulator (EP-Tracer 38; CardioTek, Maastricht-Airport, The Netherlands). From the ECG results obtained during sinus rhythm, R-R, PQ, QT, and maximal $\mathrm{T}_{\text {peak }}-\mathrm{T}_{\text {end }}$ intervals and $\mathrm{P}$ and $\mathrm{QRS}$ widths were measured. Four bipolar electrograms were recorded simultaneously from the 2 silicone strips flanking the patch.

For measurement of atrial ERP, 10 basic stimuli (S1, 400-ms interval) were applied by means of bipolar pacing at 4 times the threshold. After S1, an extra stimulus (S2) was applied starting from well within the refractory period. The S1-S2 interval was increased with 2-ms steps, and atrial ERP was defined as the longest S1-S2 interval that failed to capture.

For measurement of conduction times, the atrium was paced (400-ms interval) at the silicone strip close to the superior caval vein. Conduction time was defined as the time between stimulus artifact and corresponding atrial deflection of the electrogram recorded at the silicone patch close to the inferior caval vein, representing the conduction delay across the crista terminalis.

Inducibility of a rapid atrial response (RAR) was measured by applying $50-\mathrm{Hz}$ burst pacing during 1 second. If the burst stimulus produced a rapid irregular rhythm lasting more than 1 second, RAR was considered inducible. RAR inducibility was quantified by the percentage of RAR inductions after 20 attempts, and median RAR episode durations were calculated. 


\section{Drug Distribution Analysis}

Blood samples were regularly drawn from a peripheral vein of the hind limb through a Venflon cannula (Becton Dickinson, Franklin Lakes, NJ) up to 28 days after patch application. The blood was collected in ethylenediamine tetraacetic acid-containing Vacutainer tubes (Becton Dickinson) and centrifuged to obtain plasma that was stored at $-80^{\circ} \mathrm{C}$. At 3 hours and $1,7,14,21$, and 28 days after patch application, goats of the ALP group ( $\mathrm{n}=1$ for each time point) were killed with intravenous sodium pentobarbital, and the heart was excised. The lateral right atrium (RA) and free-wall sections $\left(4 \mathrm{~cm}^{2}\right)$ of the left atrium (LA) and both ventricles were dissected. In addition, samples of pericardium overlying the RA, pericardial fluid, the medial part of the cranial lobe of the right lung, skeletal muscle of the hind limb, and liver and abdominal fat were obtained. After immersion in liquid nitrogen for rapid freezing, tissue samples were stored at $-80^{\circ} \mathrm{C}$. For measurement of transmural drug concentration gradients, myocardial samples were freeze-microtomed parallel to the epicardial surface in slices of 500 $\mu \mathrm{m}$. High-performance liquid chromatography coupled with UV detection was used to determine amiodarone and desethylamiodarone concentrations in plasma, pericardial fluid, and tissue samples. ${ }^{11}$

\section{Statistics}

Data are expressed as means \pm standard deviations. Differences between means were calculated with a Kruskal-Wallis test for multiple comparisons. To determine the significance of differences of individual comparisons, Mann-Whitney $U$ and Wilcoxon signed rank tests were used for between-group and within-group comparisons, respectively, with post hoc Bonferroni correction.

\section{RESULTS}

\section{Feasibility and Tolerability of Patch Application}

After baseline measurements, suturing of patches to the atria was achieved within 5 minutes in each animal, without significant bleeding or changes in blood pressure. Hemodynamic and ECG parameters remained stable during the remainder of the surgical procedures. No signs of discomfort, adverse effects, or complications were observed up to 28 days after patch application. Except for a 70-ms longer $\mathrm{R}-\mathrm{R}$ interval in the ALP group than in the DFP group at day 3 after patch application, ECG parameters did not significantly differ between groups over the time period evaluated in the present study (Table 1). Gradual prolongations of R-R intervals observed in both groups during follow-up can be explained by animal habituation because similar ECG changes were observed in animals in which no patch was applied (data not shown). In all animals killed after 7 or more days, electrode strips and patches were covered by a thin $(<1 \mathrm{~mm})$ fibrous layer, without signs of pericarditis or myocarditis.

\section{Pharmacokinetic Feasibility of Atrium-Targeted Drug Delivery}

Epicardial application of amiodarone-eluting patches produced persistently higher drug concentrations in the RA than in the LA, right ventricle (RV), and left ventricle (LV; Figure 1). RA/LA, RA/RV, and RA/LV mean amiodarone concentration ratios at 3 hours after patch application were 40,67 , and 76 , respectively. These ratios increased to 1145,2247 , and 2167, respectively, at 28 days after patch application. Amiodarone concentrations reached peaks after 24 hours of patch application in all tissues studied. Cardiac tissue concentrations decreased in the next 27 days, with overall half-lives of 4 to 5 days (assuming monoexponential decrease). Peak amiodarone concentrations in extracardiac tissues were 3 to 4 orders of magnitude lower than right atrial peak drug concentrations and decreased to less than $10 \mathrm{ng} /$ $\mathrm{mL}$ (detection limit) at 21 days after patch application. For all blood samples drawn during the 28-day follow-up period, plasma amiodarone levels were less than the detection limit. Because amiodarone has a high tissue affinity, lower drug levels in plasma than in tissues are expected. These findings are also in line with the lower drug levels measured in pericardial fluid than in pericardial tissue (Figure 1,C).

TABLE 1. ECG parameters

\begin{tabular}{|c|c|c|c|c|c|c|c|c|}
\hline & & \multicolumn{7}{|c|}{ Time after patch application (d) } \\
\hline & & $\mathbf{0}$ & 1 & 3 & 7 & 14 & 21 & 28 \\
\hline \multirow[t]{2}{*}{$\mathrm{R}-\mathrm{R}$ interval (ms) } & ALP & $463 \pm 46$ & $473 \pm 44$ & $509 \pm 47^{*}$ & $546 \pm 51$ & $616 \pm 84$ & $656 \pm 82$ & $672 \pm 83$ \\
\hline & DFP & $435 \pm 55$ & $452 \pm 51$ & $439 \pm 47$ & $488 \pm 58$ & $565 \pm 56$ & $626 \pm 66$ & $648 \pm 70$ \\
\hline \multirow[t]{2}{*}{$\mathrm{P}$ width (ms) } & ALP & $64 \pm 7$ & $68 \pm 6$ & $68 \pm 7$ & $71 \pm 6$ & $73 \pm 5$ & $74 \pm 5$ & $76 \pm 5$ \\
\hline & DFP & $62 \pm 6$ & $62 \pm 7$ & $64 \pm 5$ & $66 \pm 7$ & $69 \pm 5$ & $72 \pm 4$ & $73 \pm 6$ \\
\hline \multirow[t]{2}{*}{ PQ interval (ms) } & ALP & $109 \pm 9$ & $111 \pm 10$ & $124 \pm 11$ & $136 \pm 15$ & $140 \pm 10$ & $140 \pm 8$ & $139 \pm 9$ \\
\hline & DFP & $106 \pm 13$ & $107 \pm 12$ & $113 \pm 14$ & $126 \pm 15$ & $132 \pm 10$ & $135 \pm 14$ & $136 \pm 10$ \\
\hline \multirow[t]{2}{*}{ QRS width (ms) } & ALP & $62 \pm 6$ & $64 \pm 6$ & $63 \pm 4$ & $63 \pm 5$ & $62 \pm 6$ & $62 \pm 6$ & $62 \pm 6$ \\
\hline & DFP & $62 \pm 5$ & $62 \pm 4$ & $62 \pm 4$ & $62 \pm 4$ & $63 \pm 5$ & $64 \pm 5$ & $63 \pm 5$ \\
\hline \multirow[t]{2}{*}{ QT interval (ms) } & ALP & $277 \pm 16$ & $279 \pm 18$ & $282 \pm 20$ & $290 \pm 19$ & $309 \pm 22$ & $316 \pm 20$ & $325 \pm 28$ \\
\hline & DFP & $271 \pm 22$ & $274 \pm 26$ & $262 \pm 20$ & $273 \pm 17$ & $298 \pm 19$ & $313 \pm 25$ & $322 \pm 29$ \\
\hline \multirow[t]{2}{*}{$\mathrm{T}_{\text {peak }}-\mathrm{T}_{\text {end }}(\mathrm{ms})$} & ALP & $35 \pm 4$ & $36 \pm 6$ & $37 \pm 4$ & $40 \pm 5$ & $42 \pm 6$ & $44 \pm 6$ & $47 \pm 4$ \\
\hline & DFP & $33 \pm 4$ & $34 \pm 5$ & $33 \pm 5$ & $37 \pm 6$ & $40 \pm 7$ & $44 \pm 8$ & $46 \pm 8$ \\
\hline
\end{tabular}

Presented data were obtained from electrocardiographic recordings during normal sinus rhythm. Values are presented as means \pm standard deviations. $\mathrm{n}=6$ for both the amiodarone-loaded patch $(A L P)$ and drug-free patch $(D F P)$ groups. $* P<.05$, ALP versus DFP. 

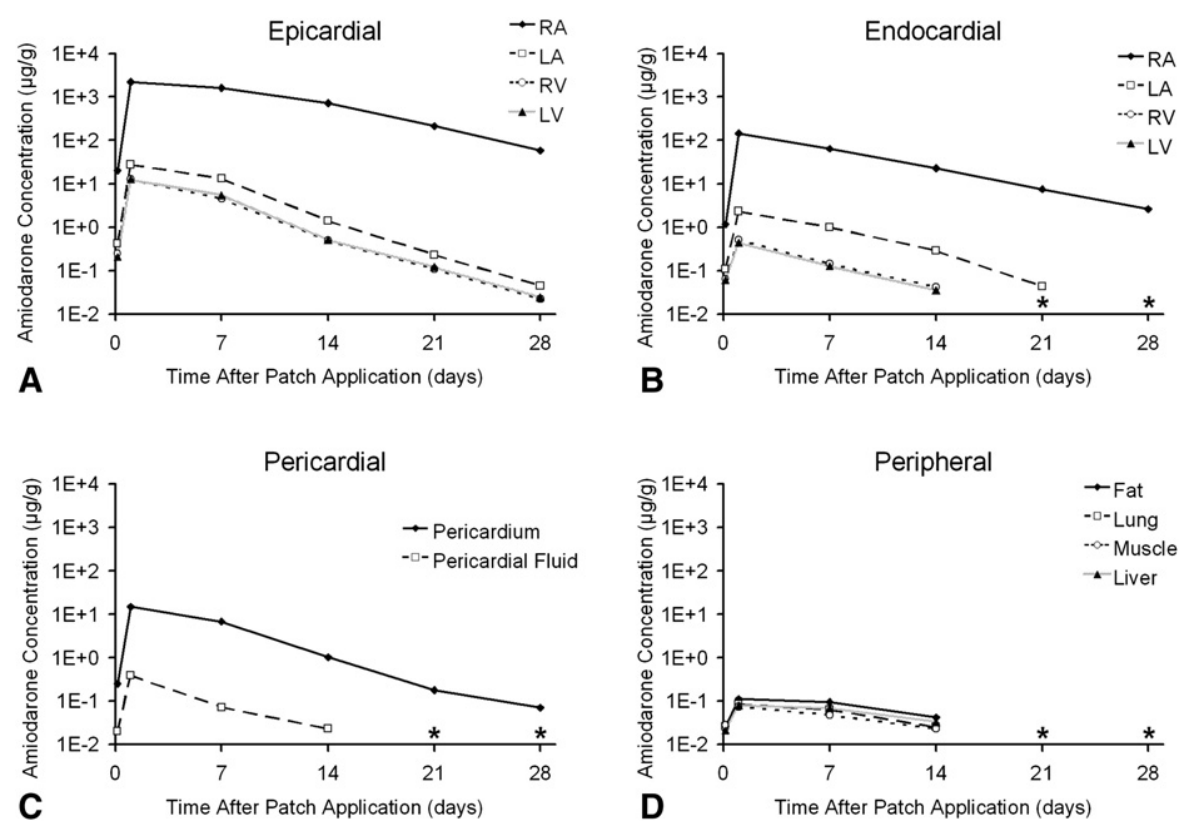

FIGURE 1. Amiodarone distribution. A, Epicardial amiodarone concentrations in the right atrium $(R A)$, left atrium $(L A)$, right ventricle $(R V)$, and left ventricle $(L V)$. B, Endocardial amiodarone concentrations in the RA, LA, RV, and LV. C, Amiodarone concentrations in pericardium and pericardial fluid. D, Amiodarone concentrations in abdominal fat, the medial part of the cranial lobe of the right lung, skeletal muscle of the hind limb, and the liver. Data were obtained 3 hours and 1, 7, 14,21, and 28 days after patch application. $\mathrm{n}=1$ for each time point. *Amiodarone concentration less than the detection limit $(<10 \mathrm{ng} / \mathrm{mL})$.

The primary active metabolite of amiodarone is desethylamiodarone. Desethylamiodarone levels in the RA were 3 orders of magnitude lower than amiodarone levels, whereas metabolite concentrations in the other heart chambers were 1 order of magnitude lower than parent drug concentrations (data not shown). Distribution of desethylamiodarone in extracardiac tissues was similar to amiodarone distribution. Plasma metabolite levels remained undetectably low during the 28-day follow-up period.

\section{Pharmacodynamic Feasibility: Antiarrhythmic Efficacy}

Effects of patch application on atrial electrophysiology are shown in Figure 2. Application of amiodarone-loaded patches produced significant increases in right atrial ERPs and conduction times over the time period evaluated in the present study. The ERP in the ALP group was maximal at $192 \pm 19 \mathrm{~ms}$ at 3 days after patch application compared with $163 \pm 10 \mathrm{~ms}$ in the DFP group. At 28 days after patch application, the ERP was still $20 \mathrm{~ms}$ higher in the ALP group. With respect to conduction times, no significant differences between groups were observed during baseline measurements. After application of amiodarone-loaded patches, conduction times tended to prolong, with mean changes ranging from $+1.3 \%$ to $+10.5 \%$, whereas a shortening was observed after application of drug-free patches, with mean changes ranging from $-5.0 \%$ to $-11.7 \%$, producing significant differences between the ALP and DFP groups (except for day 2 after patch application).

Atrial burst pacing in both groups did not produce sustained (ie, $>60$ seconds) episodes of atrial fibrillation either at baseline measurements or follow-up. Instead, RARs, typically lasting several seconds, were observed (Figure 3). RAR inducibility was significantly decreased in the ALP group compared with that seen in the DFP group from days 2 to 28 after patch application (Figure 2, C). Durations of RAR episodes tended to be longer in the DFP group, but this only reached significance in the 2 days after application of drug-free patches and during the last 2 weeks of follow-up (Figure 2,D).

\section{DISCUSSION}

The main findings of this study are that epicardial application of bilayered amiodarone-eluting patches (1) is well tolerated, (2) yields persistently higher drug concentrations in the RA than in other cardiac and extracardiac tissues (by 2-4 orders of magnitude), (3) increases ERPs and conduction times, and (4) decreases vulnerability to burst pacing-induced atrial tachyarrhythmias for at least 28 days.

Two decades ago, lidocaine-releasing polyurethane matrices were studied for the treatment of ventricular fibrillation. ${ }^{12}$ The aim of developing these drug-delivery systems was to achieve sustained therapeutic myocardial concentrations at reduced systemic drug concentrations, minimizing the risk 

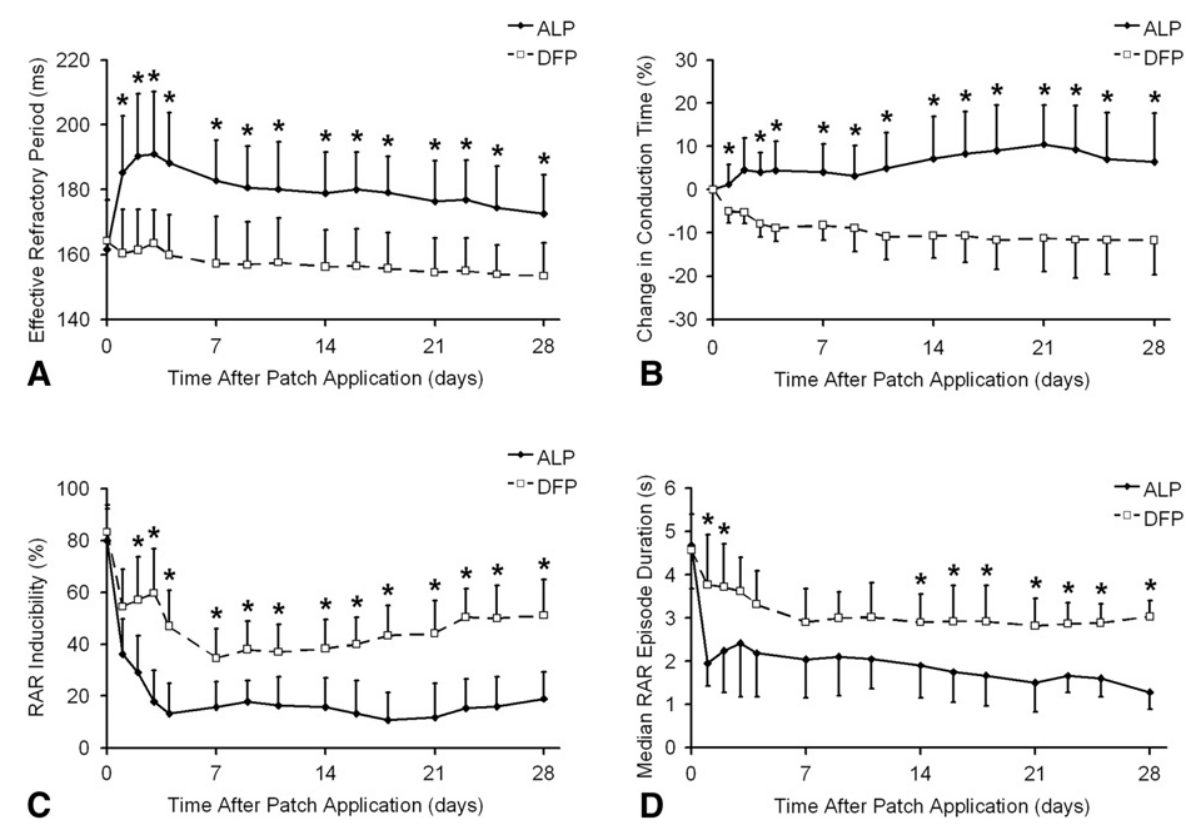

FIGURE 2. Atrial electrophysiology. A, Effective refractory period. B, Change in conduction time relative to baseline measurements. C, Rapid atrial response $(R A R)$ inducibility. D, Median RAR duration. $\mathrm{n}=6$ for both the amiodarone-loaded patch $(A L P)$ and drug-free patch $(D F P)$ groups. ${ }^{*} P<.05$.

for unwanted extracardiac effects. Polyurethane matrices were subsequently loaded with propanolol, verapamil, and ibutilide, all demonstrating successful local drug delivery for several hours in animal studies. ${ }^{13-15}$ However, polyurethane is nonbiodegradable, which limits its clinical use as a matrix for epicardial drug delivery. One study investigated the long-term effects of sotalol controlledrelease implants, including polyurethane and silicone matrices applied to the ventricular epicardium and intrapericardially infused biodegradable poly(lactic-co-glycolic acid) microspheres. ${ }^{9}$ Thirty days after poly(lactic-co-glycolic acid) microsphere delivery, atrial drug concentrations were similar to ventricular drug concentrations, whereas 60 days after silicone matrix application, sotalol levels between matrix-covered and noncovered ventricular myocardium were equal. These results indicate that the use of drug delivery systems that only consist of drug-loaded matrices do not allow targeting of one specific myocardial region. The most likely mechanism is drug release from the matrix into the pericardial fluid, which facilitates rapid drug distribution to other heart chambers. However, other transport mechanisms (eg, through subepicardial lymphatics or vascular plexi) might also contribute to pan-cardiac drug distribution.

In the present study a biodegradable bilayered patch was used, consisting of an amiodarone-loaded matrix facing the epicardium and an impermeable backing layer facing the pericardium. The latter minimizes drug release into the pericardial fluid, thereby slowing distribution to other cardiac regions and enabling longer-lasting site-specific drug delivery. Indeed, this seems to be reflected by the very high amio- darone concentrations in the epicardium of the RA compared with epicardial levels in other heart chambers up to 28 days after patch application. Regarding endocardial amiodarone concentrations, which were lower than epicardial drug concentrations, these regional differences appeared to be somewhat smaller, probably because of drug distribution through the lymphatics and blood vessels, different diffusion characteristics of the cardiac tissue layers, or both. After burst release in the first 24 hours after application, patches yielded monoexponentially decreasing amiodarone concentrations over the next 4 weeks. Epicardially applied PEG-based matrices with thioester bonds degrade within several weeks, indicating that the monoexponential decrease of drug levels was probably both diffusion and degradation mediated. ${ }^{16}$ A PLCL matrix was selected as the backing layer because this copolymer degrades much slower, on the order of 6 to 12 months, ensuring preserved impermeability during amiodarone release from the PEG-based matrix. ${ }^{17}$

Previous studies used the pericardial sac as a drug reservoir to achieve local amiodarone delivery for several hours. Intrapericardial infusion in dogs resulted in high tissue amiodarone concentrations in atria and the ventricular epicardium, whereas plasma only contained trace drug concentrations. ${ }^{6}$ In addition, atrial vulnerability to electrically induced tachyarrhythmias was decreased. In pigs it was shown that the higher atrial uptake of amiodarone, in contrast to epicardial ventricular uptake, was positively related to the intrapericardially delivered dose. ${ }^{7}$ In a recent study in goats, we observed transmural amiodarone concentration gradients with high epicardial drug levels in both atria 


\section{Amiodarone-loaded patch}

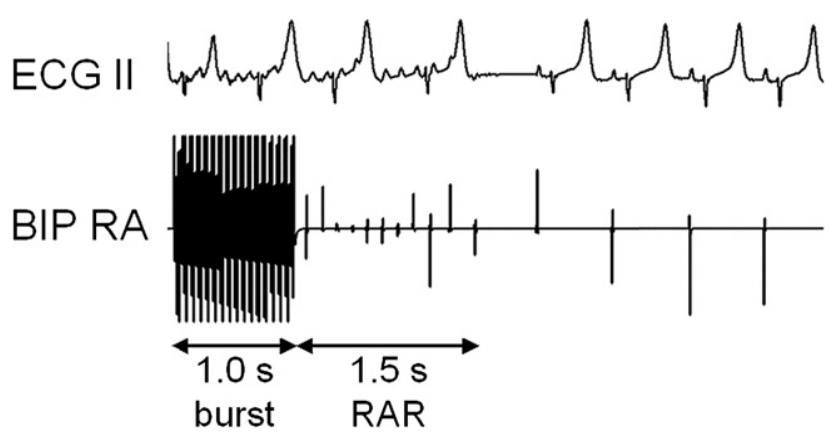

\section{Drug-free patch}

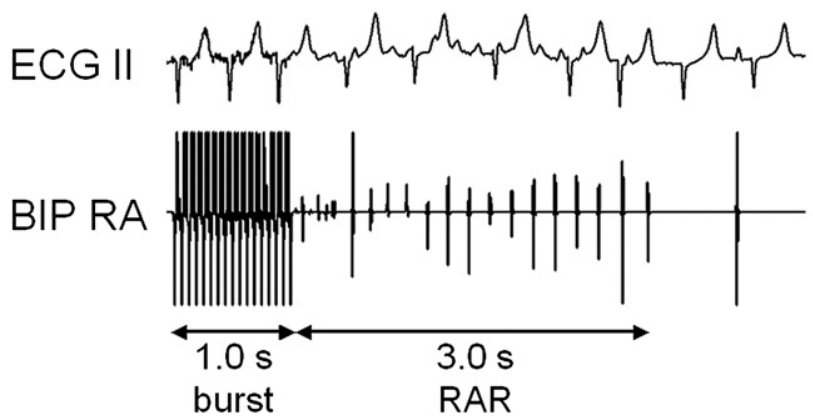

FIGURE 3. Typical responses to burst pacing 28 days after patch application. $E C G$, Electrocardiography; BIP RA, right atrial bipolar electrode; $R A R$, rapid atrial response.

after intrapericardial infusion that nevertheless produced similar epicardial and endocardial antiarrhythmic efficacy. ${ }^{8}$ Intravenous infusion of an equal amiodarone dose resulted in significantly lower antiarrhythmic efficacy both epicardially and endocardially. The present study demonstrates atrium-specific amiodarone delivery during a much longer period than seen in previous intrapericardial infusion studies. Amiodarone-loaded patches produced significant antiarrhythmic effects up to 28 days after application, whereas plasma drug levels remained less than $10 \mathrm{ng} / \mathrm{mL}$ (detection limit), which is more than 100 times lower than therapeutic plasma levels during intravenous or chronic oral amiodarone administration. Peak amiodarone concentrations in extracardiac tissues, such as fat, lung, skeletal muscle, and liver, were 2 to 4 orders of magnitude lower than levels observed during long-term oral amiodarone therapy. ${ }^{18}$

Amiodarone has multiple electrophysiologic effects, including class I, II, III, and IV antiarrhythmic actions. ${ }^{19}$ In the present study atrial ERPs were maximally prolonged in the first days after patch application, whereas effects on conduction times were maximal 2 weeks later, despite decreasing atrial amiodarone concentrations. The latter can be explained by treatment duration-dependent changes in amiodarone's ionic current blocking profile or by postoperative factors simultaneously affecting conduction time. The presence of surgical intervention-related influences on atrial electrophysiology was apparent in animals treated with drug-free patches, showing a peak of atrial arrhythmic vulnerability on days 2 and 3 after thoracotomy, which coincides with the peak incidence of POAF. ${ }^{20}$ Treatment with amiodarone-loaded patches significantly attenuated atrial vulnerability to burst pacing-induced tachyarrhythmias. Therefore efficacy of this new technique in preventing POAF is anticipated. QT intervals and maximal $T_{\text {peak }}-T_{\text {end }}$ intervals did not differ between the ALP and DFP groups, indicating low risk of drug-induced ventricular arrhythmias.

In addition to POAF, epicardial polymeric release systems have also been investigated for other clinical applications, requiring delivery of substances with a higher molecular weight than amiodarone. A PEGylated fibrin patch was used to deliver the chemokine stromal cell-derived factor-1 alpha to ventricular infarct areas of mice. ${ }^{21}$ Transmural atrial gene transfer in pigs was achieved by means of epicardial release of adenoviral vectors from a poloxamer gel. ${ }^{22}$ Growth factor-supplemented collagen matrices enhanced survival of transplanted cardiomyoblasts in ischemic rat hearts. ${ }^{23}$ These examples show the versatility of polymeric release systems, enabling advances in cardiovascular drug therapy, gene therapy, and cell therapy. ${ }^{24}$

\section{Limitations}

One important difference with human subjects is that the caprine atrial action potential is shorter. ${ }^{25}$ In addition, the atrial structure of patients with cardiovascular disease is likely to be different from the atrial structure of healthy goats. Therefore extrapolating the results of the present study to human subjects must be done with caution.

The follow-up period for this study lasted 28 days after patch application. Although this period is sufficiently long lasting for prevention of POAF, it would be interesting to study drug distribution and electrophysiologic effects for even longer periods. Patches capable of sustained delivery of amiodarone for several months might also be used in the treatment of paroxysmal atrial fibrillation. Minimally invasive access to the normal pericardial cavity might further promote the clinical applicability of epicardial polymeric release systems.

Animals in the present study did not experience sustained episodes of atrial fibrillation. Additional studies in representative animal models are required to evaluate the efficacy of local amiodarone delivery in the treatment of chronic atrial fibrillation. Epicardial and endocardial electrophysiologic mapping might provide further insight into antiarrhythmic mechanisms of epicardial amiodarone delivery.

Patches were applied only to the RA to evaluate the feasibility of sustained targeted drug delivery to a specific heart chamber. The RA was chosen to determine the negative 
chronotropic effects of patch application near the sinoatrial node. R-R intervals briefly increased after patch application, but no significant sinus bradycardia was observed. In future clinical application, patches should be applied to both the RA and LA.

Epicardially placed PEG-based biomaterials are currently used for prevention of pericardial adhesions after cardiac surgery. ${ }^{16}$ However, individual hypersensitivity to amiodarone, iodine, or patch degradation products might cause local inflammation and fibrosis after patch application. Further studies are needed to evaluate the safety of this method.

The drug-loaded matrix showed minimal adhesive properties, necessitating suturing of the patch to the atrial epicardium. Future development of a self-adhesive patch by tailoring PEG matrix characteristics could ease application and prevent needle-induced microtrauma to the atrium.

\section{CONCLUSIONS}

Atrium-targeted delivery of amiodarone is feasible through epicardial application of a bilayered patch with an amiodaroneloaded matrix facing the epicardium and an impermeable backing layer facing the pericardium. Sustained atrial antiarrhythmic effects up to 1 month after patch application can be achieved, whereas tissue drug concentrations in ventricles and extracardiac tissues remain several orders of magnitude lower than atrial drug levels. These results indicate that this method might be promising to prevent POAF with a lower risk for ventricular and systemic side effects compared with intravenous and oral amiodarone administration.

\section{References}

1. Hogue CW Jr, Creswell LL, Gutterman DD, Fleisher LA. Epidemiology, mechanisms, and risks: American College of Chest Physicians guidelines for the prevention and management of postoperative atrial fibrillation after cardiac surgery. Chest. 2005;128(suppl):9S-16S.

2. Echahidi N, Pibarot P, O'Hara G, Mathieu P. Mechanisms, prevention, and treatment of atrial fibrillation after cardiac surgery. J Am Coll Cardiol. 2008;51:793-801.

3. Fuster V, Ryden LE, Cannom DS, Crijns HJ, Curtis AB, Ellenbogen KA, et al. ACC/AHA/ESC 2006 Guidelines for the Management of Patients with Atrial Fibrillation: a report of the American College of Cardiology/American Heart Association Task Force on Practice Guidelines and the European Society of Cardiology Committee for Practice Guidelines (Writing Committee to Revise the 2001 Guidelines for the Management of Patients With Atrial Fibrillation): developed in collaboration with the European Heart Rhythm Association and the Heart Rhythm Society. Circulation. 2006;114:e257-354.

4. Camm AJ. Safety considerations in the pharmacological management of atrial fibrillation. Int J Cardiol. 2008;127:299-306.

5. Mitchell LB, Exner DV, Wyse DG, Connolly CJ, Prystai GD, Bayes AJ, et al. Prophylactic oral amiodarone for the prevention of arrhythmias that begin early after revascularization, valve replacement, or repair: PAPABEAR: a randomized controlled trial. JAMA. 2005;294:3093-100.

6. Ayers GM, Rho TH, Ben-David J, Besch HR, Zipes DP. Amiodarone instilled into the canine pericardial sac migrates transmurally to produce electrophysio- logic effects and suppress atrial fibrillation. J Cardiovasc Electrophysiol. 1996; 7:713-21

7. Darsinos JT, Karli JN, Samouilidou EC, Krumbholz B, Pistevos AC, Levis GM. Distribution of amiodarone in heart tissues following intrapericardial administration. Int J Clin Pharmacol Ther. 1999;37:301-6.

8. Bolderman RW, Hermans JJ, Rademakers LM, Jansen TS, Verheule S, van der Veen FH, et al. Intrapericardial delivery of amiodarone and sotalol: atrial transmural drug distribution and electrophysiological effects. J Cardiovasc Pharmacol. 2009;54:355-63.

9. Labhasetwar V, Underwood T, Gallagher M, Murphy G, Langberg J, Levy RJ. Sotalol controlled-release systems for arrhythmias: in vitro characterization, in vivo drug disposition, and electrophysiologic effects. J Pharm Sci. 1994;83: 156-64.

10. Dias AA, Boerakker M, Nijenhuis AJ. Polymers comprising thioester bonds. Patent Cooperation Treaty/European Patent:2006/008730.

11. Bolderman RW, Hermans JJ, Maessen JG. Determination of the class III antiarrhythmic drugs dronedarone and amiodarone, and their principal metabolites in plasma and myocardium by high-performance liquid chromatography and UVdetection. J Chromatogr B Analyt Technol Biomed Life Sci. 2009;877:1727-31.

12. Sintov A, Scott W, Dick M, Levy RJ. Cardiac controlled release for arrhythmia therapy: lidocaine-polyurethane matrix studies. J Control Release. 1988;8: 157-65.

13. Siden R, Flowers WE, Levy RJ. Epicardial propranolol administration for ventricular arrhythmias in dogs: matrix formulation and characterization. Biomaterials. 1992;13:764-70.

14. Siden R, Kadish A, Flowers W, Kutas L, Bieneman BK, DePietro J, et al. Epicardial controlled-release verapamil prevents ventricular tachycardia episodes induced by acute ischemia in a canine model. J Cardiovasc Pharmacol. 1992;19: 798-809.

15. Labhasetwar V, Underwood T, Heil RW Jr, Gallagher M, Langberg J, Levy RJ. Epicardial administration of ibutilide from polyurethane matrices: effects on defibrillation threshold and electrophysiologic parameters. J Cardiovasc Pharmacol. 1994;24:826-40.

16. Hendrikx M, Mees U, Hill AC, Egbert B, Coker GT, Estridge TD. Evaluation of a novel synthetic sealant for inhibition of cardiac adhesions and clinical experience in cardiac surgery procedures. Heart Surg Forum. 2001;4:204-10.

17. Pitt CG, Gratzl MM, Kimmel GL, Surles J, Schindler A. Aliphatic polyesters II. The degradation of poly (DL-lactide), poly (epsilon-caprolactone), and their copolymers in vivo. Biomaterials. 1981;2:215-20.

18. Adams PC, Holt DW, Storey GC, Morley AR, Callaghan J, Campbell RW. Amiodarone and its desethyl metabolite: tissue distribution and morphologic changes during long-term therapy. Circulation. 1985;72:1064-75.

19. Kodama I, Kamiya K, Toyama J. Cellular electropharmacology of amiodarone. Cardiovasc Res. 1997;35:13-29.

20. Mathew JP, Fontes ML, Tudor IC, Ramsay J, Duke P, Mazer CD, et al. A multicenter risk index for atrial fibrillation after cardiac surgery. JAMA. 2004;291: 1720-9.

21. Zhang G, Nakamura Y, Wang X, Hu Q, Suggs LJ, Zhang J. Controlled release of stromal cell-derived factor-1 alpha in situ increases c-kit+ cell homing to the infarcted heart. Tissue Eng. 2007;13:2063-71.

22. Kikuchi K, McDonald AD, Sasano T, Donahue JK. Targeted modification of atrial electrophysiology by homogeneous transmural atrial gene transfer. Circulation. 2005;111:264-70.

23. Kutschka I, Chen IY, Kofidis T, Arai T, von Degenfeld G, Sheikh AY, et al. Collagen matrices enhance survival of transplanted cardiomyoblasts and contribute to functional improvement of ischemic rat hearts. Circulation. 2006;114: I167-73.

24. Spadaccio C, Chello M, Trombetta M, Rainer A, Toyoda Y, Genovese JA. Drug releasing systems in cardiovascular tissue engineering. J Cell Mol Med. 2009;13: 422-39.

25. Franz MR, Karasik PL, Li C, Moubarak J, Chavez M. Electrical remodeling of the human atrium: similar effects in patients with chronic atrial fibrillation and atrial flutter. J Am Coll Cardiol. 1997;30:1785-92. 